\title{
DIVISIBILITY CONDITIONS ON CHARACTERISTIC NUMBERS OF STABLY SYMPLECTIC MANIFOLDS
}

\author{
D. M. SEGAL ${ }^{1}$
}

ABSTRACT. It is shown that every cohomology characteristic number of an $8 k+4$ [resp. $16 k+8$ ] dimensional stably symplectic manifold is divisible by 4 [resp. 2] and that certain characteristic numbers of 2-dimensional stably symplectic manifolds are divisible by 2 and 4 . The proofs depend on symplectic cobordism operations. Using explicit manifold constructions of Stong [5] it is shown that these results are to a large extent the best possible.

R. Stong [5] described certain "Riemann-Roch" relations on the cohomology characteristic numbers of stably symplectic manifolds. In a previous paper [4] we used these relations to compute certain differentials in the Adams spectral sequence for the 2-primary stable homotopy of $M S p$. Stong's relations give rise to extremely tedious calculations which become prohibitive after the 24 -stem and which, in any case, do not give any simple results which are valid in an infinite family of stems. In this note we show that results of P. S. Landweber [2] and simple arithmetic yield characteristic number relations relevant to the Adams spectral sequence in arbitrarily high stems.

All homology and cohomology will be with integral coefficients except as otherwise noted.

THEOREM 1. Let $f: S^{4 k} \rightarrow M S p$ be a stable map and let $s_{4 k}$ generate $H_{4 k}\left(S^{4 k}\right)$. Then

(i) If $k \equiv 1(2), f_{*}\left(s_{4 k}\right)$ is divisible by 4 .

(ii) If $k \equiv 2(4), f_{*}\left(s_{4 k}\right)$ is divisible by 2 .

COROLLARY 2. Every cohomology characteristic number of a $4 k$ dimensional stably symplectic manifold is divisible by 4 [resp. 2], if $k \equiv 1(2) \quad[r e s p . k \equiv 2(4)]$.

We will prove part (i) of Theorem 1; part (ii) may be proved by similar methods or obtained as a consequence of a theorem of $\mathrm{E}$. E.

Received by the editors March 23, 1970.

AMS 1968 subject classifications. Primary 5710, 5732.

Key words and phrases. Symplectic cobordism, stably symplectic manifolds, symplectic Pontrjagin numbers, characteristic numbers.

1 This research was supported in part by National Science Foundation Grant GP 9587. 
Floyd [1] which gives an upper bound to the image of the symplectic cobordism ring in the unoriented cobordism ring.

$H_{*}(M S p)$ may be written as the polynomial ring over the integers generated by elements $b_{i} \in H_{4 i}(M S p)$ chosen so that if $j: H P^{\infty}$ $\rightarrow B S p$ is the classifying map of the canonical quaternionic line bundle, $\phi$ the Thom isomorphism, $z$ is a generator of $H^{4}\left(H P^{\infty}\right)$ and $w_{i} \in H_{4 i}\left(H P^{\infty}\right)$ is dual to $z^{i}$, then $b_{i}=\phi j_{*} w_{i}$.

If $\omega$ is the partition $\left(n_{1}, \cdots, n_{k}\right)$, then $n(\omega)=\sum n_{i}, l(\omega)=k$.

Landweber [2] has shown that for each partition $\omega$, there is a $\operatorname{map} S(\omega): M S p \rightarrow S^{4 n(\omega)} M S p$ inducing maps (for all $m$ )

$$
\begin{aligned}
S_{*}(\omega): H_{m}(M S p) & \rightarrow H_{m-4 n(\omega)}(M S p), \\
S_{\sharp}(\omega): \pi_{m}^{S}(M S p) & \rightarrow \pi_{m-4 n(\omega)}^{S}(M S p)
\end{aligned}
$$

such that if $h_{*}$ is the Hurewicz homomorphism and $b=\sum_{i=0}^{\infty} b_{i}$, then

$$
h_{*} S_{+}(\omega)=S_{*}(\omega) h_{*}, \quad S_{*}((n)) \bar{b}=(\bar{b})^{n+1}
$$

and such that

$$
S_{*}(\omega)\left(c_{1} c_{2}\right)=\sum_{\omega=\left(\omega_{1}, \omega_{2}\right)} S_{*}\left(\omega_{1}\right)\left(c_{1}\right) S_{*}\left(\omega_{2}\right)\left(c_{2}\right) .
$$

For the general element $b \in H_{4 k}(M S p)$ we have $b=\sum_{n(\omega)=k} g_{\omega}(b) b^{\omega}$ where, if $\omega=\left(n_{1}, \cdots, n_{j}\right), b^{\omega}=\prod_{i=1}^{j} b_{n_{i}}$; the $g_{\omega}$ may be regarded as a basis for $H^{*}(M S p)$ dual to the $b^{\omega}$. We understand $g_{\omega}(b)=0$ if $b \notin H_{4 n(\omega)}(M S p)$.

Define $P(m)=\left\{\omega \mid b \in \operatorname{Im} h_{*} \Rightarrow g_{\omega}(b)\right.$ is divisible by $\left.m\right\}$.

The Adams spectral sequence for the 2-primary component of $\pi_{*}^{S}(M S p)$ shows that $(1) \in P(8)$; Proposition 3.1 of [3] shows that (2) $\in P(4)$ and $\left(2^{2}\right) \in P(2)$.

Lemma 3. $(2 k+1) \in P(4)$.

Proof. True for $k=0(P(8) \subseteq P(4))$. Assume true for $k<n$. Suppose $b \in H_{8 n+4}(M S p) \cap \operatorname{Im} h_{*}$. Let $n(\omega)=2 n+1$. Then

$$
\begin{aligned}
S_{*}((2 n-1)) b^{\omega}= & 0 \quad \text { if } \omega \neq(2 n+1),(2 n, 1),(2 n-1,2) \text { or }\left(2 n-1,1^{2}\right), \\
& S_{*}((2 n-1)) b_{2 n+1}=2 n b_{2}+\left(\begin{array}{c}
2 n \\
2
\end{array}\right) b_{1}^{2}, \\
& S_{*}((2 n-1)) b_{2 n} b_{1}=2 n b_{1}^{*}, \\
& S_{*}((2 n-1)) b_{2 n-1} b_{2}=b_{2}, \\
& S_{*}((2 n-1)) b_{2 n-1} b_{1}^{2}=b_{1}^{2},
\end{aligned}
$$


and we see that $g_{(2)}\left(S_{*}((2 n-1)) b\right)=2 n g_{(2 n+1)}(b)+g_{(2 n-1,2)}(b)$. Similarly we may argue that

$$
\begin{aligned}
g_{(2 n-1)} S_{*}((2)) b^{\omega} & =0 \quad \text { if } \omega \neq(2 n+1) \text { or }(2 n-1,2), \\
S_{*}((2)) b_{2 n+1} & =3 b_{2 n-1}+\text { other terms, } \\
S_{*}((2)) b_{2 n-1} b_{2} & =b_{2 n-1}
\end{aligned}
$$

and so

$$
g_{(2 n-1)}\left(S_{*}((2)) b\right)=3 g_{(2 n+1)}(b)+g_{(2 n-1,2)}(b) .
$$

By our inductive hypothesis and the fact that $(2) \in P(4)$ we have that 4 divides both $g_{(2)}\left(S_{*}((2 n-1)) b\right)$ and $g_{(2 n-1)}\left(S_{*}((2)) b\right)$ and so divides their difference which is $(2 n-3) g_{(2 n+1)}(b)$; since $2 n-3$ is odd, we have that 4 divides $g_{(2 n+1)}(b)$ whenever $b$ is in the image of the Hurewicz homomorphism and so $(2 n+1) \in P(4)$ completing the induction and establishing Lemma 3.

Proposition 4. If $n(\omega)=2 k+1$ then $\omega \in P(4)$.

Proof. Let $k$ be fixed. We use induction on $l(\omega)$. For $l(\omega)=1$ the proposition is just Lemma 3. Assume that the proposition is true for $l(\omega)<h$. Let $\bar{\omega}=\left(n_{1}, \cdots, n_{h}\right)$ be given, $n(\bar{\omega})=2 k+1$. Since $n(\bar{\omega})$ is odd, there must be some odd integer $d$ which occurs with odd frequency $f$ in $\bar{\omega}$. Let $\bar{\omega}^{\prime}=\bar{\omega}-\{d\}$ and let $b$ be as before an element of $H_{8 k+4}(M S p) \cap \operatorname{Im} h_{*}$. Then it is not hard to see that

$$
g_{(d)}\left(S_{*}\left(\left(\bar{\omega}^{\prime}\right)\right) b\right)=f g_{\bar{\omega}}(b)+\sum_{l(\omega)<l(\bar{\omega})} \alpha\left(\omega, \bar{\omega}, \bar{\omega}^{\prime}\right) g_{\omega}(b)
$$

where the $\alpha$ 's are integral coefficients. By Lemma 3, 4 divides the left-hand side of the above formula while by the inductive hypothesis 4 divides each summand of the second term of the right-hand side. Since $f$ is odd, 4 divides $g_{\bar{\omega}}(b)$ and so $\bar{\omega} \in P(4)$ completing the induction and establishing Proposition 4. Proposition 4 is of course merely a restatement of part (i) of Theorem 1.

Theorem 5. For $j>0,\left(2^{j}\right) \in P(4)$ and $\left(2^{j}, 2^{j}\right) \in P(2)$.

Proof. We have observed the truth of both statements for $j=1$. Assume them true for $j<k, k>1$. Suppose that $b \in H_{2^{k+2}}(M S p)$ $\cap \operatorname{Im} h_{*}$. Then

$$
g_{\left(2^{k-1}\right)}\left(S_{*}\left(\left(2^{k-1}\right)\right) b\right)=\left(2^{k-1}+1\right) g_{\left(2^{k}\right)}(b)+2 g_{\left(2^{k-1}, 2^{k-1}\right)}(b)
$$

and by induction we see that 4 divides $g_{\left(2^{k}\right)}(b) ;\left(2^{k}\right) \in P(4)$. Now suppose that $b \in H_{2}^{k+3}(M S p) \cap \operatorname{Im} h_{*}$. Then 


$$
\begin{gathered}
g_{\left(2^{k-1}, 2^{k-1}\right)}\left(S_{*}\left(\left(2^{k-1}, 2^{k-1}\right)\right) b\right)=\left(2^{k-1}+1\right)^{2} g_{\left(2^{k}, 2^{k}\right)}(b) \\
+\left(\begin{array}{c}
2^{k-1}+1 \\
2
\end{array}\right) g_{\left(3 \cdot 2^{k-1}, 2^{k-1}\right)}(b)+\left(2^{k}+2\right) g_{\left(2^{k}, 2^{k-1}, 2^{k-1}\right)}(b) \\
+6 g_{\left(2^{k-1}, 2^{k-1}, 2^{k-1}, 2^{k-1}\right)}(b) .
\end{gathered}
$$

This formula establishes that $\left(2^{k}, 2^{k}\right) \in P(2)$ under our inductive hypothesis save that for the case $k=2$ it is necessary also to observe that $(3,1) \in P(2)$ since the coefficient

$$
\left(\begin{array}{c}
2^{k-1}+1 \\
2
\end{array}\right)
$$

is then odd; that $(3,1) \in P(2)$ follows from the formula

$$
g_{(1)}\left(S_{*}((3)) b\right)=4 g_{(4)}(b)+g_{(3,1)}(b)
$$

for $b \in H_{16}(M S p)$. This completes the proof of Theorem 5 .

As in [4] (following Liulevicius [3]), let

$\operatorname{Ext}_{A}\left(H^{*}\left(M S p ; Z_{2}\right), Z_{2}\right)=\operatorname{Ext}_{B}\left(Z_{2}, Z_{2}\right) \otimes Z_{2}\left[v_{2}, v_{4}, v_{5}, \cdots, v_{i}, \cdots\right]$

$\left(i \neq 2^{a}-1\right)$. As a corollary to Theorems 1 and 5 we have

Corollary 6. No $v_{i}$ persists to $E_{\infty}$. If $i$ is odd or a power of 2 then $q_{0} v_{i}$ does not persist to $E_{\infty}$. (That is, there is no element in $E_{2}$ containing $v_{i}$ or, $i$ as indicated, $q_{0} v_{i}$ as a summand which is a permanent cycle.)

Using a construction of Stong [5] we show that Theorems 1 and 5 are to a large extent 'best possible' results:

ThEOREM 7. If $k \neq 2^{j}-1$ then $(k) \notin P(8)$. If $k$ is even and not $a$ power of 2 then $(k) \notin P(4)$.

ProOF. First, let $k$ be even and not a power of 2 . Let $a_{1}=2^{u}+1$ where $2^{u}$ is the largest power of 2 less than $2 k$; let $a_{2}=2 k+2-a_{1}$. Let $\alpha_{i}$ generate $H^{2}\left(C P^{a_{i}}\right)$. Note that $a_{i}<2 k$. In the stably symplectic $4 k+4$ manifold $C P^{a_{1}} \times C P^{a_{2}}$ we can find a stably symplectic $4 k$ manifold $M$ dual to $-\alpha^{2}, \alpha=\sum \alpha_{i}$. (Cf. Stong [5, p. 429]; Thom [6, Theorem II.1].) The total tangential symplectic Pontrjagin class of $M$ is

$$
p_{T}=\frac{\prod\left(1+\alpha_{i}^{2}\right)^{\left(a_{i}+1\right) / 2}}{1+\alpha^{2}}
$$

restricted to $M$. Now $s_{(k)}\left(p_{T}\right)=\sum\left[\left(a_{i}+1\right) / 2\right] s_{(k)}\left(1+\alpha_{i}^{2}\right)-s_{(k)}\left(1+\alpha^{2}\right)$. But $s_{(k)}\left(1+\alpha_{i}^{2}\right)=\alpha_{i}^{2 k}=0$ since $a_{i}<2 k$, so $s_{(k)}\left(p_{T}\right)=-s_{(k)}\left(1+\alpha^{2}\right)=-\alpha^{2 k}$. Then if $p_{N}$ is the total stable normal symplectic Pontrjagin class of $M$, 


$$
\begin{aligned}
s_{(k)}\left(p_{N}\right)[m] & =-\alpha^{2} s_{(k)}\left(p_{N}\right)\left[C P^{a_{1}} \times C P^{a_{2}}\right] \\
& =\alpha^{2 k+2}\left[C P^{a_{1}} \times C P^{a_{2}}\right]=\left(\begin{array}{c}
a_{1}+a_{2} \\
a_{1}
\end{array}\right) \equiv 2
\end{aligned}
$$

On the other hand $s_{(k)}\left(p_{N}\right)[M]=g_{(k)}\left(b_{M}\right)$ where $b_{M}=f_{*}^{M}\left(s_{4 k}\right), f^{M}: S^{4 k}$ $\rightarrow M S p$ being the map obtained by the Thom construction from the classifying map of the stable normal bundle of $M$. So we have $(k)$ $\notin P(4)$. If $k$ is a power of 2 the same construction but with $a_{1}=a_{2}$ $=k+1$ establishes that $(k) \notin P(8)$.

For $k$ odd Theorem 7 cannot be proven by the construction of manifolds as above. However we can obtain existence results by the same techniques that gave us the nonexistence results of Theorems 1 and 5 used in conjunction with the already proven part of Theorem 7. Given $k$ odd, $k \neq 2^{j}-1$ let $m$ be chosen to be 1,3 , or 7 so that $m-k \equiv 2$ (4) and so that $m+k$ is not a power of 2 . It is readily verified (cf. [3]) that (1), (3), (7) are all in $P(8)$. Given $b \in H_{4(m+k)}(M S p)$ $\cap \operatorname{Im} h_{*}$ we have

$$
\begin{aligned}
& g_{(m)}\left(S_{*}((k)) b\right)=(k+1) g_{(m+k)}(b)+g_{(m, k)}(b), \\
& g_{(k)}\left(S_{*}((m)) b\right)=(m+1) g_{(m+k)}(b)+g_{(m, k)}(b)
\end{aligned}
$$

and $(k) \in P(8)$ would imply that 8 divides

$$
g_{(m)}\left(S_{*}((k)) b\right)-g_{(k)}\left(S_{*}((m)) b\right)=(k-m) g_{(m+k)}(b)
$$

and so $(m+k) \in P(4)$ which we have shown to be false. We thus conclude that $(k) \notin P(8)$.

The author wishes to express his appreciation to A. Liulevicius for valuable comments concerning this paper.

\section{BIBLIOGRAPHY}

1. E. E. Floyd, Stiefel-Whitney numbers of quaternionic and related manifolds, (to appear).

2. P. S. Landweber, Cobordism operations and Hopf algebras, Trans. Amer. Math. Soc. 129 (1967), 94-110. MR $36 \# 2145$.

3. A. Liulevicius, Notes on homotopy of Thom spectra, Amer. J. Math. 86 (1964), 1-6. MR $29 \# 4060$.

4. D. M. Segal, On the symplectic cobordism ring, Comment. Math. Helv. 45 (1970), 159-169.

5. R. E. Stong, Some remarks on symplectic cobordism, Ann. of Math. (2) 86 (1967), 425-433. MR 36 \#2162.

6. R. Thom, Quelques proprietes globales des variêtés differentiables, Comment. Math. Helv. 28 (1954), 17-86. MR 15, 890.

University of Illinois at Chicago Circle, Chicago, Illinois 60680 